\title{
La calidad de vida: Análisis multidimensional
}

\author{
Ma. Guadalupe Nava Galán*
}

\section{RESUMEN}

Objetivo: Analizar el concepto de calidad de vida. Su estudio se amplió considerablemente a partir del siglo XX. El concepto se considera multidimensional dentro de una perspectiva formativa, debido a que éste cambia con las diferentes culturas, épocas y grupos sociales. Se realiza una revisión por diferentes autores.

Palabras clave: Calidad de vida, percepción, elementos objetivos-subjetivos.

\section{The quality of life: Multidimensional analysis}

\begin{abstract}
Objective: To analyze the concept of quality of life. His study was expanded considerably in the twentieth century. The concept is considered multidimensional in a formative perspective due to be changed with the different cultures, ages and social groups. A review by different authors.
\end{abstract}

Key words: Quality of life, perception, objective-subjective elements.

\section{INTRODUCCIÓN}

$\mathrm{E}$ n los últimos años, quienes se han preocupado por el interés del concepto y estudio de la calidad de vida son diversos autores, sobre todo de países desarrollados.

Los conceptos que tiene el individuo para experimentar situaciones y condiciones de su ambiente dependen de las interpretaciones, valores y de los objetivos de su entorno. ${ }^{1}$ Algunos antecedentes hacen alusión a la calidad de vida desde diferentes definiciones multidimensionales. Levy y Anderson, en 1980, refieren que "es una medida compuesta de bienestar físico, mental y social, tal y como lo percibe cada individuo o grupo, y de la felicidad, satisfacción y recompensas". ${ }^{2}$
En el mismo año, Szalai al respecto apunta que "es la evaluación subjetiva del carácter bueno o satisfactorio de la vida como un todo". ${ }^{3}$ En 1990, Celia y Tulsky la definen como "la apreciación que el paciente hace de su vida y la satisfacción con su nivel actual de funcionamiento comparado con el que percibe como posible y real". ${ }^{4}$ En 1992, Quintero refiere que es "el indicador multidimensional del bienestar material y espiritual en un marco social y cultural determinado". ${ }^{5}$ Asimismo, Felce y Perry, en 1995, la definen como la "calidad de las condiciones de vida de una persona, como la satisfacción experimentada por la persona con dichas condiciones vitales, como la combinación de componentes objetivos y subjetivos", es decir, la calidad de las condiciones de una persona junto con la satisfacción que ésta experimenta, la combinación de las condiciones de

* Maestría en Enfermería en Educación, Instituto Nacional de Neurología y Neurocirugía.

Correspondencia: Ma. Guadalupe Nava Galán. Insurgentes Sur Núm. 3877, Col. La Fama, 14269, Delegación Tlalpan, México, D.F. E-mail: rev.enf.neurol@gmail.com 
vida y la satisfacción personal ponderadas por la escala de valores, aspiraciones y expectativas personales. ${ }^{6}$

La calidad de vida es una combinación de elementos objetivos y subjetivos. ${ }^{7}$ Elementos objetivos: Bienestar material, salud objetivamente considerada, relaciones armónicas con el ambiente y la comunidad.

Elementos subjetivos: Intimidad, expresión emocional, seguridad percibida, productividad personal y salud percibida. De igual manera, la OMS define la calidad de vida como la percepción que cada individuo tiene de su posición en la vida en el contexto del sistema cultural y de valores en el que vive, en relación con sus metas, expectativas, estándares y preocupaciones. ${ }^{8}$ Éste es un concepto amplio, del que forman parte elementos como la salud física, el estado psicológico, el nivel de independencia, las relaciones sociales de la persona y su relación con el ambiente que le rodea. ${ }^{9}$ Actualmente, la disciplina de enfermería reconoce la necesidad de aplicar y desarrollar en su práctica teorías y modelos científicamente fundamentados que marquen conductas y acciones influyentes de manera general hacia conductas o estilos saludables. Se presenta la relación entre la calidad de vida y las teorías de enfermería.

\section{Teoría del autocuidado ${ }^{10}$}

Dorothea Orem presentó su teoría en la década de los cincuenta y la publicó en 1972, definiéndola como Teoría del autocuidado, la cual está compuesta por tres teorías relacionadas:

Autocuidado. Consiste en la práctica de actividades que las personas maduras inician y llevan a cabo en determinados periodos de tiempo, por sus propios medios y con el interés de mantener un funcionamiento vivo y sano para continuar con el desarrollo personal y su bienestar.

Déficit de autocuidado. Se produce cuando la relación entre las propiedades humanas de necesidad terapéutica y la capacidad de autocuidado desarrollada no son operativas o adecuadas para conocer y cubrir algunos o todos los componentes de la necesidad terapéutica de autocuidado existente. Aquí actúan los sistemas de enfermería.

Sistemas de enfermería. Son las acciones que realiza la enfermería, de acuerdo con las necesidades terapéuticas de autocuidado de sus pacientes para proteger y regular el ejercicio o desarrollo de la actividad de autocuidado de los mismos.

Esta teoría de enfermería aporta la enseñanza de manejo de signos y síntomas de los pacientes, buscando conductas de independencia y bienestar. La enfermera tiene en cuenta la cultura y habilidad de los pacientes al enseñarles y pro- porcionarles el cuidado. La independencia del paciente le genera mejor calidad de vida.

\section{Teoría de incertidumbre}

Esta teoría fue desarrollada por Mishel Merle, quien la definió como la inhabilidad del sujeto para determinar el significado de los eventos relacionados con una enfermedad; ocurre en situaciones donde debe tomar decisiones, siendo incapaz de asignar valores definitivos a objetos y eventos, y de predecir consecuencias con exactitud debido a la escasez de información y conocimiento. ${ }^{11}$ La incertidumbre es un estado cognitivo que aparece cuando un evento no es adecuadamente estructurado, debido a que la información del paciente sobre el suceso en cuestión es escasa. Los eventos que causan incertidumbre pueden ser la mayor fuente de estrés, provocando reactividad fisiológica y aumentando la emocionalidad del paciente.

La teoría es reconocida por enfermería en su práctica diaria, ayuda al enfermo y a su familia a afrontar el diagnóstico que produce estrés; el afrontamiento le va a permitir aceptar, manejar y sobreponerse a su proceso salud/enfermedad, lo cual va a contribuir en su calidad de vida.

\section{Teoría de afrontamiento}

Para Lazarus y Folkman, el individuo enfrenta el estrés a través del enjuiciamiento funcional de la actividad psíquica, y depende de fuerzas conscientes que se configuran como producto de una apreciación intuitiva de las demandas, recursos y resultados predecibles de la interacción con el medio, con modos peculiares de procesar la información y de integrar las experiencias. ${ }^{12}$

La evaluación cognitiva es el proceso que determina por qué y hasta qué punto una relación o una serie de relaciones entre el individuo y el entorno son estresantes. Éstas determinan las consecuencias en un acontecimiento dado en el individuo. La respuesta emocional y conductual del enfermo ante un acontecimiento depende de la forma en que éste lo analice.

\section{FACTORES PERSONALES QUE INFLUYEN EN LA EVALUACIÓN COGNITIVA}

Es importante tener en cuenta dos factores: los compromisos y las creencias. Los compromisos son expresiones importantes para la persona, determinan sus decisiones, alternativas que el individuo elige para conservar sus ideas y/o conseguir determinados objetivos. Tienen que ver con algo o con alguien; por lo tanto, dependen de una relación específica entre el individuo y el entorno. Las creencias son configuraciones cognitivas formadas individualmente o compartidas culturalmente, son nociones preexistenciales 
de la realidad que sirven de lente perceptual; las creencias son: la fe en Dios o en otro orden natural del universo y la esperanza; esta última busca un significado a la vida a pesar de sus experiencias dolorosas.

\section{FACTORES SITUACIONALES QUE INFLUYEN EN LA EVALUACIÓN COGNITIVA}

Es importante tener en cuenta estos tres factores: la novedad, la predictibilidad y la incertidumbre del acontecimiento. La novedad es capaz de provocar amenaza si algún aspecto de ella se ha relacionado alguna vez con el daño. La predictibilidad es un acontecimiento que ha sido estudiado, pero los hallazgos no han sido adecuados para explicar el estrés psicológico de los seres humanos, en parte porque no consideran las diferencias individuales en la evaluación y el afrontamiento. La incertidumbre del acontecimiento de la vida real suele ser estresante, pudiendo tener un efecto inmovilizador sobre procesos anticipatorios de afrontamiento, y puede provocar también confusión mental.

Esta teoría refiere que el afrontamiento es la estrategia que pone en juego al individuo ante un problema para mantener su integridad física y psicológica. La capacidad de afrontamiento de una persona depende de su trayectoria personal, de los valores y creencias que ha ido incorporando con los años. ${ }^{13}$ Hablar de un proceso de afrontamiento significa un cambio en los pensamientos y actos a medida que la interacción va desarrollándose; el afrontamiento o coping, planteado por Lazarus, se define como esfuerzos cognitivos y conductuales constantemente cambiantes que el individuo desarrolla para manejar las demandas externas y/o internas, las cuales son evaluadas como excedentes o desbordantes de sus propias capacidades de manejo para su calidad de vida.

\section{Teoría de la crisis}

Una crisis surge cuando una persona enfrenta un obstáculo importante en relación con los objetivos de vida, y es imposible superarlo mediante los métodos habituales de solución de problemas. No todas las circunstancias adversas provocan una crisis o una enfermedad, pero sí desestabilizan a la persona. ${ }^{14}$

Las crisis se producen como una respuesta emocional a una situación peligrosa. Históricamente, esta teoría remite a Freud; sus hipótesis originales establecen la relación entre situaciones vitales conscientes o inconscientes y una respuesta emocional.

En los años sesenta, Ericsson colaboró con una perspectiva evolutiva y de crecimiento en la "Teoría de la crisis". Lindemann, en colaboración con Kaplan, desarrolló esta teoría como un marco referencial para el tratamiento de situaciones de duelo.

Una crisis se define como una respuesta a eventos conflictivos, internos o externos, experimentándose un estado doloroso. En el peor de los casos, la situación de crisis no logra ser estabilizada y puede llevar a la persona a reacciones aun menos adaptativas como el suicidio, el homicidio o ambos. ${ }^{15}$ La Teoría de la crisis describe tres fases de la respuesta: 1) Fase previa a la crisis. El individuo busca mantener el equilibrio haciendo cambios físicos y psicosociales en el contexto de los sucesos normales de vida. 2) La fase de crisis se caracteriza por la desorganización; se hacen intentos para resolver el problema, que pueden resultar o no. La opción es aliviar la angustia mediante la evaluación médica inmediata y la participación activa en el plan de tratamiento. 3) En la fase posterior a la crisis, también surgen varias posibilidades.

\section{Modelo de creencias en salud}

Es uno de los modelos más utilizados en promoción de la salud; incluye un importante componente cognitivo-perceptivo. Fue originalmente desarrollado en los años cincuenta por un grupo de especialistas en psicología encabezados por Hochbaum; éste se adapta para explicar una variedad de conductas como la respuesta individual ante ciertos síntomas de enfermedad, el cumplimiento del paciente con los tratamientos y las recomendaciones médicas, la práctica de autoexámenes exploratorios, entre otros. ${ }^{16}$

También se basa en teorías del aprendizaje, en particular la conductista, que defiende que todo tipo de comportamiento puede ser reducido a relaciones estímulo-respuesta elementales y justificado por sus inmediatas consecuencias; justifica el comportamiento como el resultado de procesos mentales en los que el sujeto otorga cierto valor a las consecuencias de su acción y aprecia la probabilidad de que ésta produzca el resultado deseado. Los defensores de ambas teorías consideran que el reforzamiento es un importante factor condicionante del comportamiento, lo sitúan influenciando las expectativas o hipótesis, en lugar del comportamiento en sí.

Es una teoría construida con la valoración subjetiva de una determinada expectativa. En términos de salud, el valor es el deseo de evitar la enfermedad o el padecimiento, y la expectativa es la creencia en que una acción posible de realizar prevendrá o mejorará el proceso.

El modelo de creencias en salud se basa en tres premisas: 1) la creencia -o percepción- de que un determinado problema es importante o suficientemente grave como para tenerlo en consideración; 2) la creencia -o percepción- de que uno es vulnerable a ese problema, y 3) la creencia -o percepción- 
de que la acción a tomar producirá un beneficio a un costo personal aceptable.

En la investigación de Sanhueza, los factores del modelo que determinan la conducta de salud son de dos tipos: la percepción de amenazas sobre la propia salud y las creencias de los individuos sobre la posibilidad de reducir esas amenazas. Una variable que completa el modelo es la presencia de estímulos internos o externos que se constituyen en claves para actuar. Una clave interna puede ser el síntoma de una enfermedad, mientras que una clave externa puede ser una campaña acerca de la promoción en salud o las interacciones sociales con amigos afectados por alguna enfermedad.

En las últimas décadas, existe una extraordinaria labor para ampliar la información. Ésta considera aspectos filosóficos, metodológicos y teóricos alrededor del concepto de calidad de vida, de acuerdo con teorías que hacen que la vida sea mejor, considerando perspectivas de bienestar mental y espiritual, calidad de las relaciones interpersonales, funcionamiento e integridad del cuerpo, posesión de aspectos materiales, entre otros. ${ }^{17,18}$

Sin embargo, Brock conceptualizó la calidad de vida como "una vida buena", fundamentándola en tres grandes teorías: la hedonista, la relacionada con la satisfacción de las preferencias y la que por virtud de los ideales se realice una buena vida. ${ }^{19}$

Teoría hedonista: Se apoya en las experiencias conscientes como amor, placer, felicidad, disfrute; éstas acompañan la satisfacción de los deseos.

Teoría de la satisfacción de los deseos: Se toma como experiencia consciente o como objetos; lo bueno para una persona es que obtenga lo que más desea o prefiere. Para varios autores, las dos categorías anteriores comprenden lo que se ha denominado como bienestar.

Teoría de una buena vida: En esta teoría se deben realizar ideales específicos, explícitamente normativos como la autodeterminación o autonomía. El elemento formal para estos componentes son vectores y ponderaciones independientes para que puedan enmarcarse como una descripción general en la calidad de vida. ${ }^{20}$ Se enfoca principalmente sobre las capacidades para hacer ciertas cosas básicas. La capacidad refleja la libertad de una persona para elegir entre diferentes formas de vida.

También existen diversas teorías cognitivas que consideran la calidad de vida.

Teoría de la comparación social: Se refiere a la tendencia a relacionar sus propias características actuales o potenciales como: la salud, perspectivas futuras, la forma de afrontar alguna situación; la comparación influye en cambios de humor, autoevaluación, satisfacción personal y estrategias para afrontar alguna situación. Es la que mejor plantea aspectos subjetivos en el concepto de calidad de vida. Esta teoría es bastante real en el sentido de que dentro de las sociedades la comparación existe en la mayoría de los ámbitos. ${ }^{21}$

Teoría de la subjetividad: Plantea que la comprensión de comparaciones son parte de la persona; la forma en cómo construye el mundo es la que le va a dar las pautas para recibir la información de las demás personas; de acuerdo con la imagen que tenga de sí mismo va a compararse con realidades "mejores" o "peores". Todo esto depende de la propia concepción de su realidad, de su vida personal y de sus vivencias.

Teoría de la observación de la propia vida y de la propia realidad: En esta teoría, se llega a una conclusión luego de hacer una comparación con otras personas o grupos, lo cual es parte de un proceso evidentemente subjetivo, ya sea cognitivo o emocional. A partir de las comparaciones, la persona considerará el propio bienestar subjetivo o calidad de vida. ${ }^{22}$

En cuanto a las teorías que no consideran la subjetividad dentro de sus planteamientos, se consideran:

La Teoría de la actividad: Es amplia en su concepción, en el sentido que no es específica por parte de la persona; al momento de realizar la actividad, lo que otorga es bienestar subjetivo. Con base en esto, se considera que es bastante reduccionista y deja de lado elementos relevantes al momento de hablar de las personas que se encuentran insertas en una sociedad. Ésta incluye: factores personales, las diferentes concepciones de actividad, las historias de cada persona, es decir, los componentes que conforman la subjetividad de la persona. ${ }^{23}$

Se distinguen dos componentes en la evaluación de la CV: la satisfacción, como un componente cognitivo de bienestar y la felicidad, que es el componente afectivo.

Es importante destacar que la calidad de vida considera que el bienestar está fuertemente ligado a los ingresos y comúnmente a la presencia de medios adecuados. Recientemente se le atribuye mayor importancia, como la cualidad para obtener satisfacción a través del disfrute de los recursos disponibles y no sólo de su mera posesión. Diversos autores asumen una relación causa-efecto entre los recursos y las condiciones de vida: mientras más y mejores recursos se tenga, mayor es la probabilidad de una buena calidad de vida, para ello se requiere de diferentes factores: ${ }^{24}$

Factores materiales: Son los recursos que uno tiene, tales como: ingresos disponibles, posición en el mercado de trabajo, salud y nivel de educación, entre otros.

Factores ambientales: Son las características de los habitantes/comunidad que pueden influir en la calidad de vida, como la presencia y acceso a servicios, grado de seguridad y criminalidad, transporte y movilización, habilidad para servirse de las nuevas tecnologías que hacen la vida más 
simple. También, las características del hogar son relevantes en determinar la calidad de las condiciones de vida.

Factores de relacionamiento: Se incluyen las relaciones con la familia, los amigos y las redes sociales. La integración a organizaciones sociales y religiosas, el tiempo libre y el rol social después del retiro de la actividad económica son factores que pueden afectar la calidad de vida en edades avanzadas. Cuando la familia juega un rol central en la vida de las personas adultas mayores, los amigos, vecinos y otras redes de apoyo son importantes. ${ }^{25}$

Factores sociales y calidad de vida: La calidad de vida recibe la influencia de factores como: el empleo, la vivienda, el acceso a servicios públicos, las comunicaciones, la urbanización, la criminalidad, la contaminación del ambiente y otros, que conforman el entorno social y que influyen sobre el desarrollo humano de una comunidad.

\section{CLASIFICACIÓN DE LA CALIDAD DE VIDA}

\section{Calidad de vida y felicidad}

Es un concepto que sostiene que se debe medir en términos de la utilidad, ya sea en felicidad o satisfacción de deseos o preferencias. Epicuro, el gran doctor en felicidad, según Schopenhauer, dividió las necesidades humanas en tres clases: las naturales y necesarias son las que no son satisfechas y producen dolor, incluyen el alimento y el vestido, y son relativamente fáciles de satisfacer; las naturales pero no necesarias, tal como la satisfacción sexual, más difícil de satisfacer, y las que no son naturales ni necesarias, que incluyen el lujo, la abundancia, el fausto y el esplendor, siendo su satisfacción muy difícil.

El estudio de la felicidad ha sido durante mucho tiempo el dominio de los filósofos moralistas, aunque a lo largo de las últimas décadas también los científicos sociales se han interesado por el tema. La distribución de la felicidad considera categorías sociales. Ideológicamente, se inspira en el credo utilitarista de que el fin último de la política debería ser el promover "la mayor felicidad para el mayor número de personas" siendo los beneficios de la felicidad el criterio de la "utilidad de todas las acciones". Esta filosofía moral del siglo XIX es la base ideológica del estado de bienestar del siglo XX que fue el propulsor de la investigación sobre calidad de vida, según lo señalan Ovalle y Martínez. ${ }^{26}$

Durante siglos, el término "felicidad" se ha utilizado como muletilla para todos los significados de calidad de vida. En filosofía, prevalecieron los dos primeros significados: en la filosofía social el significado de "buenas condiciones de vida" (felicidad como buena sociedad) y en la filosofía moral, el significado de buena acción (felicidad como virtud).
En la ciencia social prevalece la palabra "felicidad" como disfrute subjetivo de la vida. ${ }^{27}$

El enfoque utilitarista que asimila la calidad de vida a la de felicidad queda sujeto a controversia, por las muchas y diversas actividades que hacen a una vida humana floreciente. La controversia remite a la filosofía de la ciencia, política del conocimiento, modelo de sociedad y concepto de ser humano como actor individual y social. Como puede apreciarse, la definición de calidad de vida humana implica una antropología filosófica. La formulación y la utilización del concepto de calidad de vida humana debe implicar, además, una filosofía social elaborada a partir de la perspectiva cultural y política de América Latina en el contexto de las relaciones de interdependencia internacional.

Es posible pensar en diferentes tipos o dimensiones de calidad de vida humana; este autor reflexiona y hace un enfoque dialéctico a partir de dos planos analíticos: calidad instrumental frente a calidad sustantiva y calidad individual frente a calidad colectiva. Carl Gustav Jung, psiquiatra suizo, desarrolló una teoría acerca del concepto del inconsciente colectivo. Estableció que existe un lenguaje común a los seres humanos de todos los tiempos y lugares del mundo que serían los símbolos primitivos, a través de los cuales se expresa un contenido de la psiquis que está más allá de la razón. El inconsciente colectivo estaría vinculado con los instintos, que serían necesidades fisiológicas manifestadas en fantasías presentes frecuentemente por medio de imágenes simbólicas, por lo cual sería todo menos un sistema aislado y personal. ${ }^{28}$

\section{Calidad de vida instrumental}

La calidad instrumental refleja una condición utilitaria, extrínseca y formal; significa perfección tecnocrática y sofisticación de medios independientemente de su contenido político y su relevancia cultural. Se identifica con la competición desenfrenada e interesada, que tiende a acumular bienes materiales, independientemente de los valores éticos establecidos colectivamente por la población.

Su criterio está orientado por los destinos de la humanidad, significa que se corre el riesgo de llevar el mundo a la degradación ecológica, destrucción de los lazos sociales y del ser humano. Quizá sea ésta la postura de las últimas décadas de la sociedad, en su desenfreno por vivir rodeada de lujos y confort. ${ }^{29}$

\section{Calidad de vida sustantiva}

Refleja una condición ética e intrínseca del ser humano como actor individual y social, políticamente engranado en la sociedad; significa calidad de vida política creada 
históricamente por la población en su propia comunidad, da prioridad a la definición y a la satisfacción de las necesidades básicas y a la promoción colectiva de la población en su medio cultural.

Se preocupa por la distribución equitativa de los bienes materiales y no materiales producidos por la población. Se identifica con la promoción de la participación de los individuos y grupos en las decisiones que afectan al bien común. Respeta las instituciones, los valores endógenos y promueve la autonomía cultural. ${ }^{30}$

Se identifican tres connotaciones en el concepto calidad de vida: calidad del entorno en el cual uno vive, usado por los ecologistas en sus luchas contra la degradación del medio ambiente y por los sociólogos cuando reivindican mejoras sociales, representando las condiciones externas para una vida llevadera; calidad de acción, que tiene que ver con la capacidad de la gente para enfrentarse a la vida; sería ésta la capacidad de vivir ampliamente utilizada por las profesiones terapéuticas como las de Ciencias de la Salud, y la calidad del resultado, que requiere condiciones previas. Las dos anteriores implican la capacidad para lograr una buena vida, que para ser buena tiene que ser de nuestro agrado, haciendo evidente el componente subjetivo.

\section{Calidad de vida y ética de las capacidades}

Es posible considerar la vida que lleva una persona como una combinación de varios quehaceres y seres, a los que genéricamente se les puede llamar funcionamientos, siendo cada uno de éstos las facetas en los que un ser humano puede tener interés o se puede ver realizado (salud, alimentación, educación, trabajo, diversión, placer, política, relaciones, entre otros). Está íntimamente ligada a las capacidades de una persona, como: la libertad que un ser humano tiene para elegir la clase de vida que quiere para sí mismo.

Se recurre, por influencia de Nussbaum, a Aristóteles para explicitar los fundamentos filosóficos de la ética de las capacidades: "Las relaciones conceptuales más importantes parecen ser las vinculadas con la noción aristotélica del bien humano. La explicación está vinculada con la necesidad de establecer primero la función del hombre y luego proceder a explorar la vida en el sentido de actividad." ${ }^{31}$

Propone Nussbaum, ${ }^{32}$ desde la perspectiva aristotélica, determinar "una lista de funcionamientos que constituyen una buena vida humana", ciertas características de nuestra común humanidad, aunque se experimenten de manera diferente en distintas culturas: la mortalidad, el cuerpo humano, la capacidad de placer y dolor, la capacidad cognitiva, la razón práctica, el desarrollo infantil temprano, la afiliación o sociabilidad, el humor y sentido lúdico. De entre ellas, hay dos: la afiliación (o sociabilidad) y la razón práctica, las cuales desempeñan un papel arquitectónico en la vida humana, al permear y organizar las demás funciones, determinando lo que debe entenderse como "naturaleza humana". Nussbaum encuentra en Aristóteles una filosofía esencialista, una visión de la vida humana que tiene ciertos rasgos centrales definitorios: "propiedades esenciales". Sin esta consideración, se carece de una base objetiva suficiente para dar cuenta de la justicia social y fundar una ética global. Sin embargo, Sen sostiene que lo decisivo no son los medios como tales, sino tener libertad con la que se pueda llevar el tipo de vida que merece ser valorada. Esta "capacidad" de una persona se refiere a la libertad sustantiva para conseguir distintas combinaciones de funciones para lograr el estilo de vida que uno quiera. Lo primordial no son las funciones (las cosas que se hacen), sino las capacidades (las oportunidades reales).

Conill pone de relieve la diferencia entre dos centros de atención axiológicos: 1) el de los "resultados funcionales", que pueden ser los mismos y 2) el del "conjunto de capacidades", el poder efectivo de decidir (el poder disponer). Éste constituye el nivel más radical del sentido de "capacidad" y de las funciones; considera que se trata del sentido kantiano de la libertad. De ahí, Sen ha ido formulando su enfoque de las capacidades cada vez más insistentemente como "la perspectiva de la libertad". La vida buena se presenta en versión de vida libre (frente a la pobreza y la tiranía), ofreciendo una base para la dignidad humana.

Por tanto, la noción de "capacidad" no sólo está relacionada con la dynamis aristotélica, sino con el modo moderno de entender las capacidades, más cercano al enfoque ilustrado de Smith, Kant y Marx.

Esta nueva noción -moderna- de "capacidad", constitutiva de la capacidad dinámica, se convierte en una fuente de valor, en un poder valorizado, consistente en otorgar dignidad a la persona humana por tratarse de algo que (objetivamente) es fin en sí y no mero medio (subjetivamente) para otra cosa. Se puede agregar que la calidad de vida puede ser un ideal objeto de búsqueda continua por parte del ser humano, que simultáneamente se encuentra en permanente cambio, puesto que representaría un equilibrio dinámico entre lo que se es, lo que se tiene y lo que uno representa individualmente y como ser gregario, haciendo uso de las categorías desglosadas por Schopenhauer.

Sin duda, se debe tener una concepción del ser humano integral, en el que confluye lo biológico, psicológico, social, ético, político, cultural, histórico, económico, espiritual y estético, sin que ningún aspecto sea más relevante que otro para determinar la calidad de vida. Para algunos, la clave está en lo económico; sin embargo, otros consideran que lo cardinal está en el ser y el hacer, en esa capacidad de elegir que postulan Nussbaum y Sen que permitiría el logro de 
buenos fines (una vida buena y floreciente) a través de buenos medios. Es decir, la integración de la reflexión y la acción, de la contemplación y el sentido de la vida, para entender por qué se hace más fácil el hacer frente a los demás, empleando la filosofía como terapia y reivindicando la máxima de Nietzsche: "Podremos manejar cualquier cosa con tan sólo preguntarse un por qué", y cómo esto tiene sentido constructivo y edificante para la propia vida y la de los demás. Nussbaum, desde su perspectiva antropológica y aristotélica, propone una lista de capacidades humanas "esenciales". Éstas se estructuran en dos umbrales:

a) Un primer umbral está compuesto por funciones humanas básicas. Incluye nuestra mortalidad y nuestra consciencia, la capacidad para experimentar placer y dolor, la capacidad cognitiva, un desarrollo infantil temprano y la dependencia del adulto, la razón práctica, la planificación y manejo de nuestra propia vida, el sentimiento de filiación con otros seres humanos, la relación con otras especies, la naturaleza, el humor, el juego y la individualidad.

b) Sobre este umbral, Nussbaum distingue uno superior de corte ético que se caracteriza en forma tan reducida, a pesar de que juzguemos qué es una vida humana. En la versión formalista y universal de la moralidad, la Filosofía defiende desde Kant una Ética de la justicia; propone el paso al nivel preconvencional (lógica hedonista-instrumental); el nivel convencional postula la autonomía como criterio, así como el cambio desde un enfoque instrumental hacia un enfoque de derechos. El nivel postconvencional es el nivel superior, se caracteriza por la pretensión universal, por los principios abstractos: la asunción de rol permite que el sujeto se ponga en los zapatos de otro para decidir, que asuma como suyas las reglas morales, las legales y los principios éticos universales a través de los cuales se defienden los derechos éticos para toda la humanidad, derechos que todos los acuerdos sociales deberían recoger. En este sentido, constituye el respeto de la persona en sí misma y de los estilos de vida que lleva a cabo. ${ }^{33}$

\section{ESTILOS DE VIDA}

Ruiz Olabuenaga en 1989 refiere que el estilo de vida es un concepto amplio; sin embargo, Simmel fue el primero en utilizarlo como "estilización", término que le da sentido a la necesidad que poseen los individuos y las clases sociales como "estilizar la vida". El estilo de vida se caracteriza actualmente por la iniciativa que tiene el individuo de recrear su cosmos social, personal y diferenciado; conlleva un conjunto de prácticas, hábitos, valores, actitudes, tendencias, consumos y formas vitales. Se ha identificado el estilo de vida principalmente con la salud en un plano biológico, pero no la salud como bienestar biopsicosocial, espiritual o como componente de la calidad de vida.

Ética del desarrollo. Se hace una reflexión sobre los fines y medios que acompañan a los cambios socioeconómicos en los países y regiones pobres. El desarrollo humano debe tener algún contenido sustantivo, existiendo controversia acerca de cuáles contenidos morales han de ser tomados en cuenta por la utilidad con la satisfacción de preferencias o bienes primarios sociales. Rawls se refiere a éstos como: ingresos y libertad negativa. Bauer considera las necesidades humanas básicas; Streeten refiere importante la autonomía; O'Neill, las capacidades y funcionamientos valiosos, y Sen, los derechos.

Los deberes de las personas, deben fluir desde la idea de que todos los humanos tienen el derecho a un mínimo nivel de bienestar. Las sugerencias de la ética son las capacidades que cada quien tiene y defiende con responsabilidad gubernamental; capacitar a cada uno para ser protagonista y avanzar hacia un nivel de suficiencia respecto a los funcionamientos valiosos que tiene cada quien.

\section{CONCLUSIÓN}

La ética del desarrollo aplica la sabiduría para fortalecer el bienestar humano y el desarrollo internacional a través de un diálogo interdisciplinario e intercultural. Debido a la necesidad de unificar criterios éticos sobre la definición de calidad de vida, la Organización Mundial de la Salud en 1994 propuso un consenso: "Percepción del individuo sobre su posición en la vida, en el contexto de la cultura y sistema de valores en el cual él vive, y en relación con sus objetivos, expectativas, estándares e intereses." 34

En este sentido, se ha señalado que la calidad de vida tiene que ver con las condiciones que favorezcan modos de vida que privilegien el ser más sobre el tener más, puesto que está íntimamente relacionada con el sentido que se tenga de uno mismo como perspectiva individual y con un sentimiento de realización existencial. ${ }^{35}$

\section{PERSPECTIVA INDIVIDUAL}

Esta concepción realza el carácter subjetivo de la calidad de vida, puesto que la misma se construye histórica y culturalmente con valores sujetos al desarrollo de cada época y sociedad, y su forma particular de mirar el mundo con subjetividad. ${ }^{36}$ Por ello, la calidad de vida está condicionada por el medio cultural y depende estrictamente del conjunto de valores de los individuos y de los grupos sociales. Para Gracia, la calidad de vida se estructura en dos niveles: uno público y otro privado. Cada individuo define individual y socialmente su propio sistema de valores, su concepto 
de vida y de calidad y, por tanto, de felicidad. Desde ahí establece el propio proyecto de vida. Esto es lo que hoy se llama "ética de máximos", que en Bioética expresa los principios de autonomía y beneficencia. Al mismo tiempo, los miembros de una sociedad deben contratar, mediante el procedimiento de la voluntad general, un conjunto de valores que sean respetados por todos, aun obligadamente. En el nivel de la ética pública y la definición general de calidad de vida que realiza la sociedad se expresa en forma de leyes. Este nivel se denomina "ética de mínimos" de una sociedad. En Bioética se habla en este nivel de principios de no maleficencia y de justicia.

De tal manera que en la calidad de vida de los seres humanos están implícitos componentes muy personales y otros gregarios, cuya armonización es nuestra responsabilidad como sujetos morales hermanados con el mundo, con el que se interactúa racional y afectivamente como integrantes de una comunión hombre-naturaleza, en el ejercicio progresivo de la autonomía libre-relacionada, en reciprocidad de deberes y derechos.

Existen tópicos que se consideran sustantivos para evaluar una vida como poseedora de calidad objetiva; éstos son fundamentalmente la satisfacción de aspectos básicos como lo planteó Maslow en su pirámide que por jerarquías de necesidades indica que conforme se satisfacen las necesidades básicas, los humanos desarrollan necesidades y deseos más altos. Se postula la existencia de necesidades de orden alto que se satisfacen a manera externa de autorrealización y autoestima, y social y de orden bajo, como son las necesidades fisiológicas y de seguridad, satisfechas internamente. Es por ello que se trata de una categoría multidimensional que combate el concepto del ser humano lineal, uniforme y que obliga a desplegar la creatividad para aprehender la diversidad humana; su percepción depende en gran parte de la concepción del mundo que tiene el sujeto en particular, la interpretación y valoración que le da a lo que tiene, vive y espera.

Algunos autores proponen un modelo de calidad de vida, considerando que existe responsabilidad de ciertas necesidades básicas para la supervivencia; por ello se concibe al ser humano inmerso en la sociedad, enmarcado en un lugar, en una cultura y con elementos que regulan e incluso limitan la cosmovisión del sujeto.

\section{BIBLIOGRAFÍA}

1. Ardila R. Calidad de vida: una definición integradora. Revista Latinoamericana de Psicología 35 (2): 161-164.

2. Levy L, Anderson L. La tensión psicosocial, población, ambiente y calidad de vida. Editorial. El Manual Moderno México 1980: 7.

3. Szalai A. The meaning of comparative research on the quality of life. In: Szalai A, Andrew FM. The quality of life: Comparative studies. Londres Inglaterra; 1980.
4. Celia DF, Tulsky DS. Measuring the quality of life today: methodological aspects. Oncology 1990; 4: 29-38.

5. Quintero G. Comunicación personal. Barcelona, España; 2001: 45-47.

6. Borthwick-Duffy SA. Quality of life and quality of care in mental retardation. In: Rowitz L (Editor). Mental retardation in the year 2000 Berlin: Springer-Verlag; 1992: 52-56.

7. Op. cit. Ardila R. p. 162.

8. Organización Mundial de la Salud (OMS). Foro Mundial de la Salud. Ginebra; 1996.

9. Borthwick-Duffy SA. Quality of life and quality of care in mental retardation. In: Rowitz L (Editor). Mental retardation in the year 2000 Berlin: Springer-Verlag; 1992: 52-56. (Repetida: 6)

10. Marriner A, Raile M. Modelos y teorías en enfermería. $5^{\text {a }}$ ed.: Elsevier Science, Madrid; 2003.

11. Mishel M. Reconceptualization of the Uncertainty in Illness. Theory. Journal of Nursing Scolarship 1990; 22 (4): 256-257.

12. Lazarus R, Folkman S. Estrés y procesos cognitivos. Martínez Roca, Barcelona; 1996.

13. Montoya R. El afrontamiento y la pérdida: visiones positivas de la labor de un cuidador: Nicolás Robles Gravi. Index Enferm Digital [en Internet] 2004 [Acceso: 04/10/2004]: 44-45. Disponible en: http://www.indexf-en fermería/44revista/44artículo-56-59.php

14. Martínez GC. Ilustración clínica de la técnica de intervención en crisis. Rev de Psiquiatría 1999; 15 (3): 169-174.

15. Op. cit. Martínez GC.

16. Soto F, Lacaste J, Papenfess R et al. El modelo de creencias en salud. Un enfoque teórico para la prevención de SIDA. Rev Esp Salud Pública 1997; 71 (4): 335-341.

17. Parfit D. "Reasons and persons". Citado por Dan Brock. Medidas de la calidad de vida en el cuidado de la salud y la ética médica. En: Nussbaum MC, Sen A (Compiladores). La calidad de vida. The United Nations University, Fondo de Cultura Económica, México; 1998: 136.

18. Dijkers M. Quality of life after spinal cord injury: A meta-analysis of the effects of disablement components. Spinal Cord 1997; 35: 829-840.

19. Brock D. Medidas de calidad de vida en el cuidado de la salud y la ética médica. En: Nussbaum MC, Sen A (Compiladores). La calidad de vida. México: The United Nations University, Fondo de Cultura Económica; 1998: 135-181.

20. Sen A. Capacidad y bienestar. En: Nussbaum MC, Sen A, compiladores. La calidad de vida. México: The United Nations University, Fondo de Cultura Económica; 1998: 54-83.

21. Clark L. Social cognition and health psychology. In: Wyer RS Jr, Srull TK. Edit Handbook of social cognition. Vol. 2. Applications, pp. 239-288.

22. Camargo M. Calidad de vida y capacidades humanas. Rev Geo Venez 1999; 40 (2): 247-58. Disponible en: http://www.saber.ula. $\mathrm{ve/db/ssaber/Edocs/pubelectronicas/revistageografica/vol40} \mathrm{num2/}$ articulo40-2-4.pdf

23. Camargo M. Calidad de vida y capacidades humanas. Rev Geo Venez 1999; 40 (2): 247-58. Disponible en: http://www.saber.ula.ve/db/ssaber/ Edocs/pubelectronicas/revistageografica/vol40 num2/articulo40-2-4.pdf

24. Sen A. Capacidad y Bienestar. En: Nussbaum M, Sen A. La Calidad de Vida. México: Fondo de Cultura Económica. 1998: 74-75.

25. Sen A. Capacidad y Bienestar. En: Nussbaum M, Sen A. La Calidad de Vida. México: Fondo de Cultura Económica. 1998: 74-75.

26. Ovalle O, Martínez J. La calidad de vida y la felicidad. Escuela de Economía Internacional, UACH. En: Contribuciones a la Economía, diciembre 2006. Disponible en: http://www.eumed. net/ ce/2006/oojm.htm

27. Shopenhauer A. Arte del buen vivir. $19^{\mathrm{a}}$ Edición.: Editorial EDAF S.A. Madrid; 2006.

28. Ovalle O, Martínez J. La calidad de vida y la felicidad. Escuela de Economía Internacional, UACH. En: Contribuciones a la Economía, diciembre 2006. Disponible en: http://www.eumed. net/ce/2006/oojm. htm

29. Bueno S. Calidad de vida humana como criterio epistemológico. En: Educación, administración y calidad de vida. Aula XXI, Editorial 
Santillana, Argentina, 1990: 9-13. Disponible en: http://www.santillana. com.ar/02/xtextos/0301.asp?level=\&mat=eteorico \&sec $=1$

30. Bueno S. Calidad de vida humana como criterio epistemológico. En: Educación, administración y calidad de vida. Aula XXI, Editorial Santillana, Argentina, 1990: 9-13. Disponible en: http://www.santillana. com.ar/02/xtextos/0301.asp?level=\&mat=eteorico \&sec $=1$

31. Nussbaum M, Sen A. La calidad de vida. Fondo de Cultura Económica, México; 1998: 34-36.

32. Op. cit. Nussbaum M, p. 38.

33. Bueno S. Calidad de vida humana como criterio epistemológico. En: Educación, administración y calidad de vida. Aula XXI,
Editorial Santillana, Argentina, 1990: 9-13. Disponible en: http:// www.santillana.com.ar/02/xtextos/0301.asp?level=\&mat=eteorico $\& \sec =1$

34. Bueno S. Calidad de vida humana como criterio epistemológico. En: Educación, administración y calidad de vida. Aula XXI, Editorial Santillana, Argentina, 1990: 9-13. Disponible en: http://www.santillana. com.ar/02/xtextos/0301.asp?level=\&mat=eteorico \&sec $=1$

35. Maslow A. Una teoría de la motivación humana. 1943. Revisión psicológica, 50, 370-96. Junio de 2001. Disponible en: http://psychclassics. yorku.ca/Maslow/motivation.htm

36. Op. cit. Maslow A, p. 96. 